\title{
Weakly-supervised convolutional neural networks of renal tumor segmentation in abdominal CTA images
}

\author{
Guanyu Yang ${ }^{1,2^{*}}$, Chuanxia Wang ${ }^{1}$, Jian Yang ${ }^{3}$, Yang Chen ${ }^{1,2}$, Lijun Tang ${ }^{4}$, Pengfei Shao ${ }^{5}$, Jean-Louis Dillenseger ${ }^{6,2}$,
} Huazhong Shu ${ }^{1,2}$ and Limin Luo $0^{1,2}$

\begin{abstract}
Background: Renal cancer is one of the 10 most common cancers in human beings. The laparoscopic partial nephrectomy (LPN) is an effective way to treat renal cancer. Localization and delineation of the renal tumor from pre-operative CT Angiography (CTA) is an important step for LPN surgery planning. Recently, with the development of the technique of deep learning, deep neural networks can be trained to provide accurate pixel-wise renal tumor segmentation in CTA images. However, constructing the training dataset with a large amount of pixel-wise annotations is a time-consuming task for the radiologists. Therefore, weakly-supervised approaches attract more interest in research.
\end{abstract}

Methods: In this paper, we proposed a novel weakly-supervised convolutional neural network (CNN) for renal tumor segmentation. A three-stage framework was introduced to train the CNN with the weak annotations of renal tumors, i.e. the bounding boxes of renal tumors. The framework includes pseudo masks generation, group and weighted training phases. Clinical abdominal CT angiographic images of 200 patients were applied to perform the evaluation.

Results: Extensive experimental results show that the proposed method achieves a higher dice coefficient (DSC) of 0.826 than the other two existing weakly-supervised deep neural networks. Furthermore, the segmentation performance is close to the fully supervised deep CNN.

Conclusions: The proposed strategy improves not only the efficiency of network training but also the precision of the segmentation.

Keywords: Weakly-supervised, Renal tumor segmentation, Bounding box, Convolutional neural network

\section{Background}

Renal cancer is one of the ten most common cancers in human beings. The minimally invasive laparoscopic partial nephrectomy (LPN) is now increasingly used to treat the renal cancer [1]. In the clinical practice,

\footnotetext{
* Correspondence: gyyang1980@qq.com; yang.list@seu.edu.cn

'LIST, Key Laboratory of Computer Network and Information Integration, Southeast University, Ministry of Education, Nanjing, China

${ }^{2}$ Centre de Recherche en Information Biomédicale Sino-Français (CRIBs), Rennes, France

Full list of author information is available at the end of the article
}

some anatomical information such as the location and the size of the renal tumor is very important for the LPN surgery planning. However, manual delineation of the contours of the renal tumor and kidney in the pre-operative CT images including more than 200 slices is a time-consuming work. In recent years, deep neural networks have been the widely used for organ and lesion segmentation in medical images [2]. However, fully-supervised deep neural networks were trained by a large number of training images with

(C) The Author(s). 2020 Open Access This article is licensed under a Creative Commons Attribution 4.0 International License, which permits use, sharing, adaptation, distribution and reproduction in any medium or format, as long as you give appropriate credit to the original author(s) and the source, provide a link to the Creative Commons licence, and indicate if changes were made. The images or other third party material in this article are included in the article's Creative Commons licence, unless indicated otherwise in a credit line to the material. If material is not included in the article's Creative Commons licence and your intended use is not permitted by statutory regulation or exceeds the permitted use, you will need to obtain permission directly from the copyright holder. To view a copy of this licence, visit http://creativecommons.org/licenses/by/4.0/ The Creative Commons Public Domain Dedication waiver (http://creativecommons.org/publicdomain/zero/1.0/) applies to the data made available in this article, unless otherwise stated in a credit line to the data. 
pixel-wise labels, which take a considerable time for radiologists to build. Thus, weakly supervised approaches attract more interest, especially for medical image segmentation.

In recent years, several weakly-supervised $\mathrm{CNNs}$ have been developed for semantic segmentation in natural images. According to the weak annotations used for CNN training, these approaches can be divided into four main categories: bounding box [3-6], scribble $[7,8]$, points $[9,10]$ and image-level labels [11-17]. However, as far as we know, there are only a few weakly-supervised methods reported for the segmentation tasks in medical images. DeepCut [18] adopted an iterative optimization method to train CNNs for brain and lung segmentation with the bounding-box labels which are determined by two corner coordinates, and the target object is inside the bounding box. In another weakly-supervised scenario [19], fetal brain MR images were segmented using a fully convolutional network (FCN) trained by superpixel annotations [20] which refer to an irregular region composed of adjacent pixels with similar texture, color, brightness or other features. Kervadec et al. [21] conducted a size loss on CNN, which was used to obtain the segmentation of different organs from the scribbled annotations which annotate different areas and their classes. These weakly learned-based methods have achieved comparable accuracy on normal organs but have not yet been applied to lesions. The approaches for renal tumor segmentation are mainly based on traditional methods such as level-set [22], SVM [23] and fully-supervised deep neural networks $[24,25]$. To the best of our knowledge, there is no weakly-supervised deep learning technique reported for renal tumor segmentation.
As shown in Fig. 1, the precise segmentation of renal tumors is a challenging task because of the large variation of the size, location, intensity and image texture of renal tumors in CTA images. For example, small tumors are often overlooked since they are difficult to be distinguished from the normal tissue, as displayed in Fig. 1(b). Different pathological types of renal tumors show varied intensities and textures which increases the difficulty of segmentation [26]. Thus, the segmentation of renal tumors by a weakly-supervised method is still an open problem.

In this paper, bounding boxes of renal tumors are provided as weak annotations to train a $\mathrm{CNN}$ which can generate pixel-wise segmentation of renal tumors. Compared to the other types of annotations, the bounding box is a simple way to be defined by radiologists [27]. The main contributions of this paper are as follows:

(1) To the best of our knowledge, we proposed a weakly-supervised $\mathrm{CNN}$ for renal tumor segmentation for the first time.

(2) The proposed method can accomplish network training faster and overcome the undersegmentation problem compared with the iterative training strategy usually adopted by the other weakly-supervised CNNs [18, 28].

(3) The experimental results of a 200-patients clinical dataset with different pathological types of renal tumors show that the CNN trained by our method can provide precise renal tumor segmentation.

The remaining paper is organized as follows: Materials section describes the datasets used in this paper. In Methods section the method is introduced in detail.

\section{A}
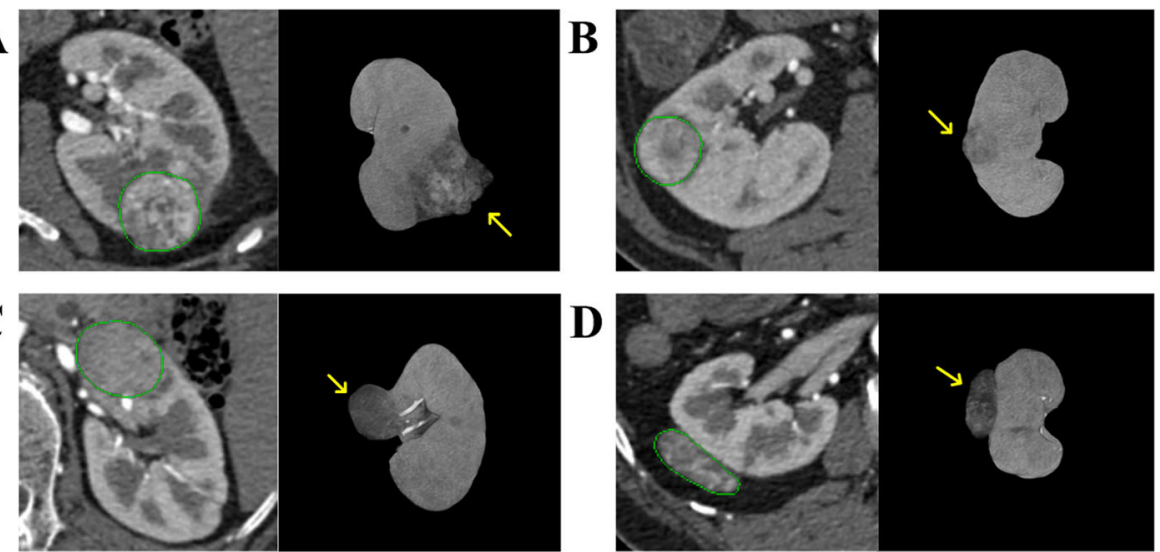

Fig. 1 Four contrast-enhanced CT images of different pathological renal tumors. The tumors are marked by yellow arrows in 3D views. The manual contours of the renal tumors delineated by a radiologist are displayed in 2D slices. The pathological subtypes of the renal tumors are clear cell renal cell carcinoma (RCC) in (a) and (b), chromophobe RCC in (c) and angiomyolipoma in (d) 
Experimental results are summarized in Results section. We give extra discussion in Discussion section, a conclusion in Conclusion section and abbreviations section. The last section is the declarations of this paper.

\section{Materials}

The pre-operative CT images of 200 patients who underwent an LPN surgery were included in this study. The CT images were generated on a Siemens dualsource 64-slice CT scanner. The contrast media was injected during the CT image acquisition. The study was already approved by the institutional review board of Nanjing Medical University. Two scan phases including arterial and excretion phases were performed for data acquisition. In this paper, $\mathrm{CT}$ images acquired in arterial phase were used for training and testing. The arterial scan was triggered by the bolus tracking technique after $100 \mathrm{ml}$ of contrast injection (Ultravist 370, Schering) in the antecubital vein at a velocity of $5 \mathrm{ml} / \mathrm{s}$. Bolus tracking used for timing and scanning was started automatically $6 \mathrm{~s}$ after contrast enhancement reached $250 \mathrm{HU}$ in a region of interest (ROI) placed in the descending aorta. The pixel size of these CT images is between $0.56 \mathrm{~mm}^{2}$ to $0.74 \mathrm{~mm}^{2}$. The slice thickness and the spacing in $\mathrm{z}-$ direction were fixed at $0.75 \mathrm{~mm}$ and $0.5 \mathrm{~mm}$ respectively. After LPN surgery, pathological tests were performed to examine the pathological types of renal tumors. Five types of renal tumors were included in this study, i.e. clear cell RCC (172 patients), chromophobe RCC (4 patients), papillary RCC (6 patients), oncocytoma (6 patients) and angiomyolipoma (12 patients). The volume of the renal tumors' ranges from $12.21 \mathrm{ml}$ to $159.67 \mathrm{ml}$ and the mean volume is $42.58 \mathrm{ml}$.

As shown in Fig. 2(a), each original CT image was resampled to an isotropic volume with the size of axial slice equal to $512 * 512$. The original CT image contained the entire abdomen, whereas only the area of the kidney needed to be considered in this experiment. Thus, the kidneys in the images were firstly segmented by the multi-atlas-based method [29] to define the ROIs of kidneys as shown in Fig. 2(b). The multi-atlas-based method just produce initial segmentation of kidneys, two radiologists checked the contours of kidneys and corrected them if necessary. The contours of tumors were drawn manually by one radiologist with 7-years' experience and checked by another radiologist with 15-years' experience in the cross-sectional slices. However, the pixel-wise masks were only used for bounding boxes generation and testing dataset evaluation. Among 200-patient images, 120 patients were selected to build the training dataset and the other 80 patients were used as the testing dataset.

\section{Methods}

We train our proposed method via bounding boxes of renal tumors to obtain pixel-wise segmentation. Thus, a pre-processing step is performed before the training procedure of weakly-supervised model. In Pre-processing section, the pre-processing including normalization and bounding box generation is briefly introduced. Then the proposed weakly-supervised method is illustrated in detail in Weakly supervised segmentation from bounding box Section. Finally, the parameters of training are explained in Training section.

\section{Pre-processing Normalization}

As is done in other studies, original CT images should be normalized before fed into the neural network. Due to the existence of bones, contrast media and air in the intestinal tract, CT values in the abdominal CT image or extracted ROIs can range from
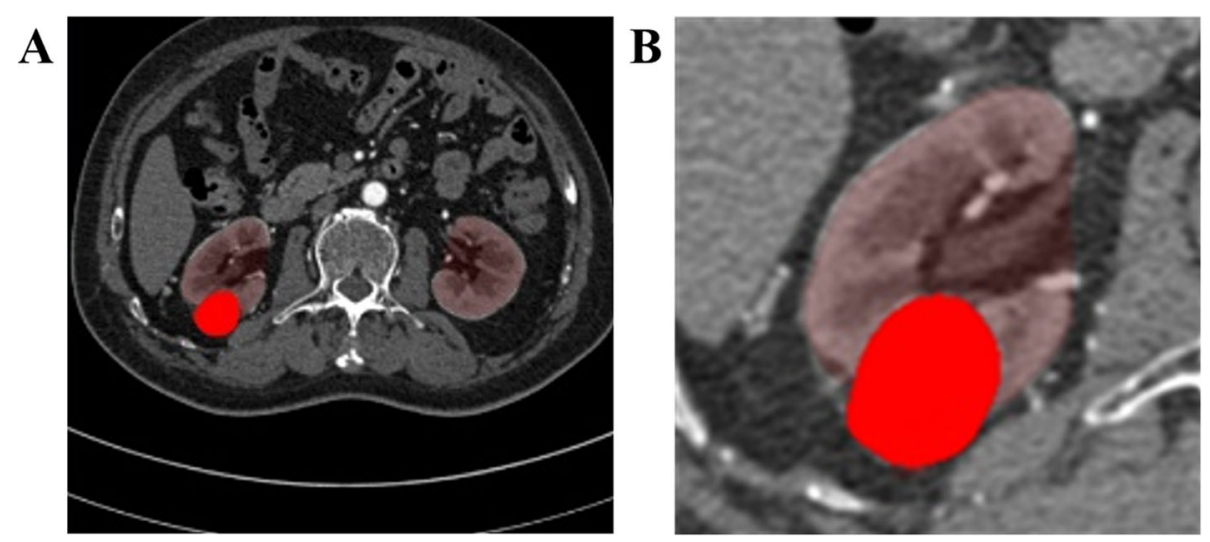

Fig. 2 a The original image with labeled kidney and renal tumor. The region in red represents renal tumor. $\mathbf{b}$ The cropped original image with the label for renal tumor segmentation 
$-1000 \mathrm{HU}$ to more than $800 \mathrm{HU}$. Thus, Hounsfield values were clipped to a range of -200 to $500 \mathrm{HU}$. After thresholding, the pixel values in all images are normalized to $0 \sim 1$ by Min-Max Normalization:

$$
X^{\prime}=\frac{X-X_{\min }}{X_{\max }-X_{\min }}
$$

\section{Bounding box generation}

In this paper, bounding boxes are generated by ground truth of renal tumors. As shown in Fig. 3, the bounding box of ground truth is shown in the dotted line. The parameter $d$ in pixel represents the margin added to the bounding box in our experiment to generate different types of weak annotations. In addition, the reference labels of renal tumors in the training dataset were only used to generate bounding boxes and not used for $\mathrm{CNN}$ training, and the reference labels in the testing dataset were used for quantitative evaluation.

The bounding boxes with different margins are defined according to the ground truth and used as weak annotations for $\mathrm{CNN}$ training. We set $d$ to be 0,5 and 10 pixels (Fig. 4(a)-(c)) in our study to simulate the manual weak annotations by radiologists. If the bounding boxes with margin $d$ are beyond the range of images, it will be limited in the region of images. As shown in Fig. 4, the comparison of bounding boxes with different margin values is given.

\section{Weakly supervised segmentation from bounding box}

Three main steps are included in the proposed method as shown in Fig. 5. Firstly, we get pseudo masks from bounding boxes by convolutional conditional random fields (ConvCRFs) [30]. Then, in the group training stage, several CNNs are trained by using pseudo masks. Fusion masks and voxel-wise weight map are generated based on the predictions of the CNNs trained in this stage. In the last stage of weighted training, the final $\mathrm{CNN}$ is trained by fusion masks and voxel-wise weighted cross-entropy (VWCE) loss function. These three main stages are described in the following Pseudo masks generation, Group training and fusion mask generation and Training with VWCE loss sections respectively.

\section{Pseudo masks generation}

As adopted by other methods [3, 18], the pseudo masks of renal tumors are generated from bounding boxes as initialization for $\mathrm{CNN}$ model training. The quality of pseudo masks influences the performance

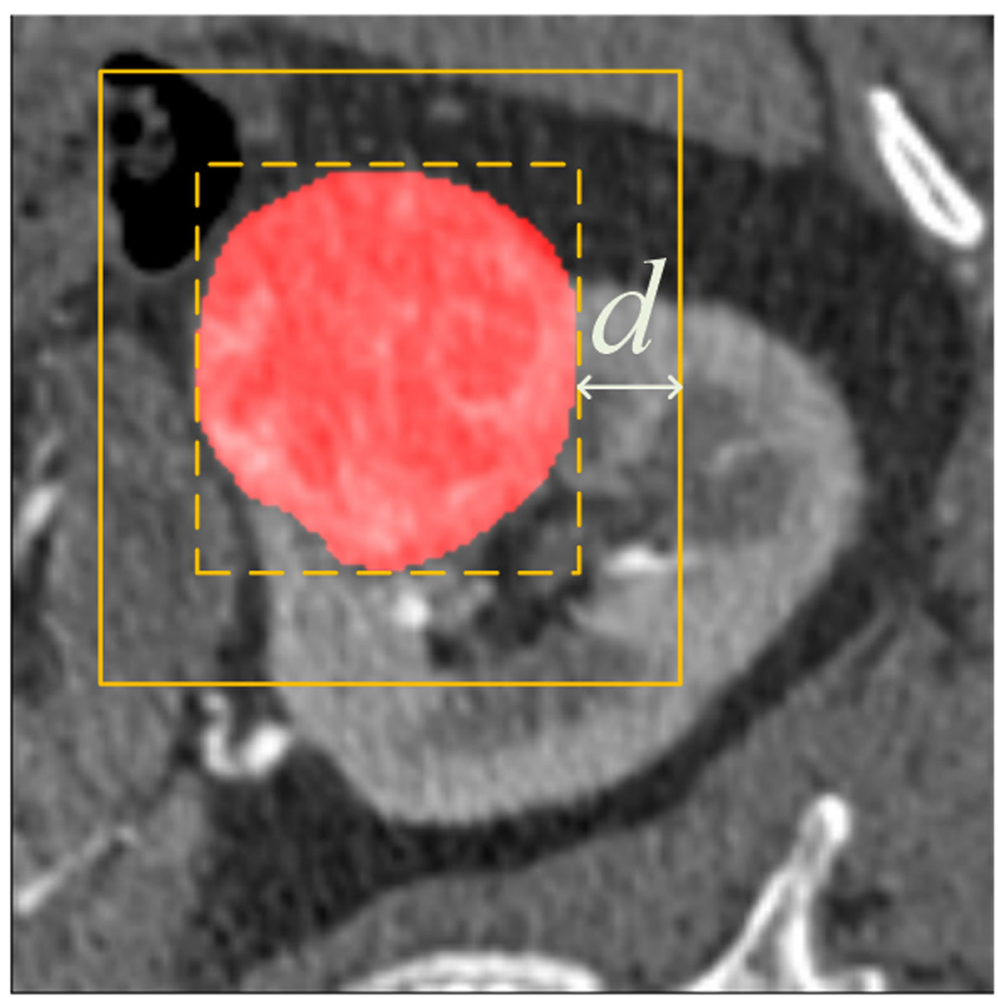

Fig. 3 The bounding box with margin $d$ is defined as weak annotations according to the label of renal tumors 

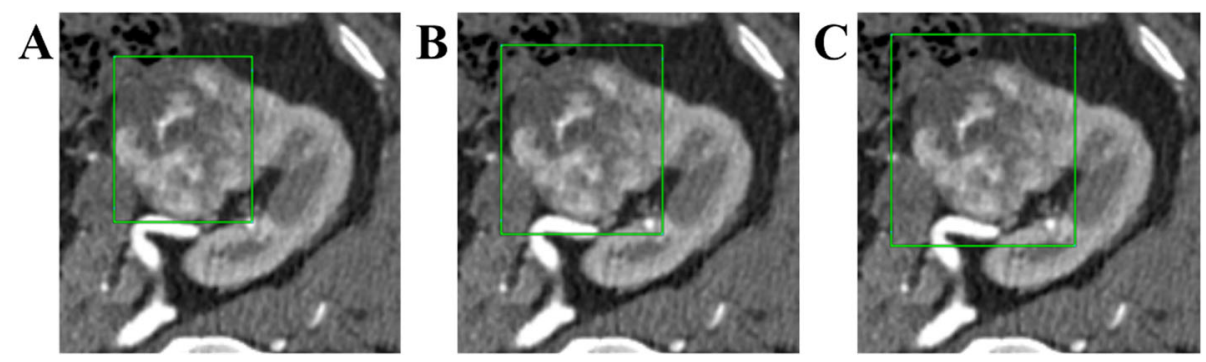

Fig. 4 Comparison of bounding boxes with different margins. The 2D image is the maximum slice. Contours in green correspond to bounding boxes

of CNN. Inspired by fully connected conditional random fields (CRFs) [31], this problem can be regarded as maximum a posteriori (MAP) inference in a CRF defined over pixels [5]. The CRF potentials take advantage of the context between pixels and encourage consistency between similar pixels. Suppose an image $X=\left\{x_{1} \ldots x_{N}\right\}$ and corresponding voxel-wise label $Y=\left\{y_{1} \ldots y_{N}\right\}$, here $y_{i} \in\{0,1\} . y_{i}=0$ means $x_{i}$ is located outside the bounding box, while $y_{i}=1$ means $x_{i}$ is located inside the bounding box. The CRF conforms to the Gibbs distribution. Then, the Gibbs energy can be defined as:

$$
E(X)=\sum_{i} U\left(y_{i}\right)+\sum_{i, j} P\left(y_{i}, y_{j}\right)
$$

where the first term is unary potential, representing the energy of assigning class $y_{i}$ to the pixel $x_{i}$, which is given by the bounding box. The latter term represents the pairwise potential, which is used to represent the energy of two pixels $x_{i}$ and $x_{j}$ in the image whose label are assigned to $y_{i}$ and $y_{j}$ respectively. In the fully connected CRFs, the pairwise potential function is defined as follows:

$$
P\left(y_{i}, y_{j}\right)=\mu\left(y_{i}, y_{j}\right) \sum_{i \neq j \leq N} w \cdot g\left(f_{i}, f_{j}\right)
$$

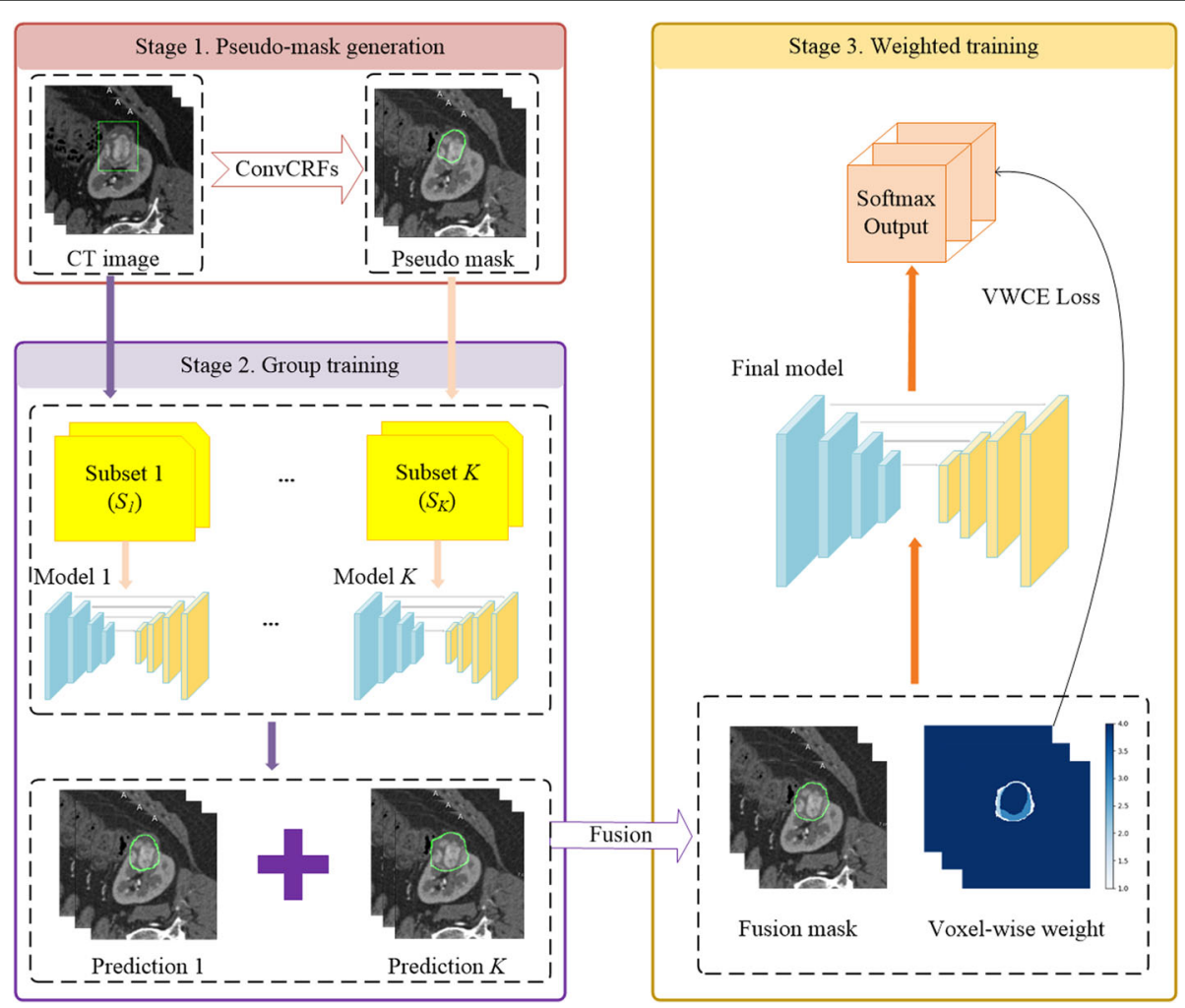

Fig. 5 An overview of the proposed weakly-supervised method 
where $w$ is a learnable parameter, $g$ is the gaussian kernel defined by feature vectors $f$ and $\mu$ is a label compatibility function.

However, because the volumetric image was used in our study, the computation of fully connected CRFs has high time complexity. Thus, inspired by Teichmann et al. [30], ConvCRFs were used for our pseudo masks generation. ConvCRFs adds the assumption of conditional independence into fully connected CRFs. Here, the matrix of gaussian kernel changes to:

$$
g\left(f_{i}, f_{j}\right)=\exp \left(-\sum_{i \neq j \leq D} \frac{f_{i}-f_{j}}{2 \theta^{2}}\right)
$$

where $\theta$ is a learnable parameter and $D$ is the Manhattan distance between pixels $x_{i}$ and $x_{j}$, the pairwise energy is zero when the Manhattan distance exceeds $D$. The complexity of pairwise potential is simplified when conditional independence is added.

The merged kernel matrix $G$ is calculated by $\Sigma w$. $g$, and the inference result is $\Sigma G \cdot X$ which is similar to convolutions of CNNs. This assumption makes it possible to reformulate the inference in terms of convolutions in CRF, which can carry out efficient GPU calculation and complete feature learning. Thus, we can quickly get pseudo masks of renal tumors by minimizing the object function defined by Eq. (2).

\section{Group training and fusion mask generation}

Once we have generated pseudo masks of renal tumors, these masks are fed into $\mathrm{CNN}$ as weak labels for parameter learning. Most of weakly supervised segmentation methods used iterative training $[5,7]$ to optimize the accuracy of the weak labels from coarse to fine. However, the preliminary results showed that this iterative strategy is hard to improve the accuracy of pseudo masks due to the difficulties of the renal tumor segmentation mentioned before. To overcome this problem, we proposed a new $\mathrm{CNN}$ training strategy instead of iterative training method.

In the group training stage, we have input images $\left\{X_{1} \ldots X_{M}\right\}$ and pseudo masks $\left\{I_{1} \ldots I_{M}\right\}$. The input training dataset is divided into $K$ subsets $\left\{S_{1} \ldots S_{K}\right\}$. For each subset $S_{k}$, a CNN $f\left(X ; \theta_{k}\right), X \in S_{k}$ with parameter $\theta_{k}$ is trained. In total, we can get $K$ CNNs trained in this stage. After that, for each image $X_{m}$, we can get $K$ predictions $\left\{P_{m}^{1} \ldots P_{m}^{K}\right\}$ of renal tumors by these CNN models. We denote that $P_{m}^{k}=f\left(X_{m} ; \theta_{k}\right\}$. Pseudo code of group training is shown in Algorithm 1.

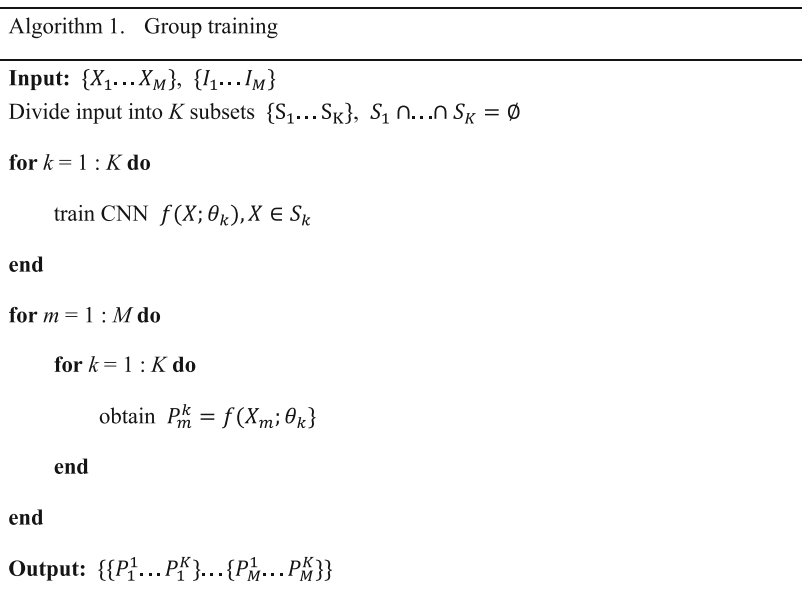

One thing worth to be mentioned is that one image in the training dataset is used to train only one CNN model in this stage. Once $K \mathrm{CNN}$ models are trained successfully, all the images in the training dataset will be used to test each $C N N$ model and obtain $K$ results for prediction. Thus, the proposed group training strategy can ameliorate the overfitting of the model. In order to alleviate the under-segmentation in the $K$ predictions, a mask image is generated by fusing these predictions. The fusion mask is defined as follows:

$$
F M_{m}=\operatorname{ConvCRFs}\left(P M_{m} \cup P_{m}^{1} \cup \ldots \cup P_{m}^{K}\right)
$$

where $F M$ indicates the fusion masks, and $P M$ indicates pseudo masks generated in Pseudo masks generation section. The ConvCRFs is adopted to refine the union of all prediction masks. The outputs of ConvCRFs will be used as the new weak labels for the next weighted training stage. In addition, a weight map is generated simultaneously which is defined as follows:

$$
v_{m}=P M_{m}+P_{m}^{1}+\ldots+P_{m}^{K}, v[v=0]=K+1
$$

When the predicted label of a voxel is renal tumor in one prediction result, its $v_{m}$ will be an integer within the range of 1 to $K+1$. When $v_{m}$ is equal to 0 , its value will be reset to $K+1$ to represent the weight of background.

\section{Training with VWCE loss}

After Section Pseudo masks generation and Group training and fusion mask generation, the fusion masks of training dataset are generated for the final $\mathrm{CNN}$ model training in this stage. Only the final $\mathrm{CNN}$ model will be used for testing dataset evaluation. In this stage, we train the $\mathrm{CNN}$ on the whole training dataset with the fusion masks. In addition, a new voxel-wise weighted cross-entropy (VWCE) loss function is 
designed to constrain the $\mathrm{CNN}$ training procedure. The traditional cross-entropy loss is defined as follows:

$$
L_{C E}=-\frac{1}{M} \sum_{m \in M} \sum_{c \in C} F M_{m, c} \log f\left(X_{m, c} ; \theta\right)
$$

where $F M$ are fusion masks defined in Eq. (5), $f(X ; \theta)$ are the outputs of $\mathrm{CNN}, M$ represents the number of samples and $C$ represents the number of classes. In Eq. (7), pixels belonging to different classes have equal weight. In the case of unbalanced datasets, [32] proposed weighted cross-entropy loss defined as follows:

$$
L_{W C E}=-\frac{1}{M} \sum_{m \in M} \sum_{c \in C} w_{c} F M_{m, c} \log f\left(X_{m, c} ; \theta\right)
$$

where, $w_{c}$ represents the weight of class $c$. Considering the weak annotations used in the training procedure, the voxel-wise weight map generated in the previous stage represents the probability of the predicted class given in the fusion mask. Thus, the voxel-wise weights obtained in Eq. (6) are introduced into Eq. (8) which is defined as follows:

$$
L_{V W C E}=-\frac{1}{M} \sum_{m \in M} v_{m} \sum_{c \in C} w_{c} F M_{m, c} \log f\left(X_{m, c} ; \theta\right)
$$

Finally, we conduct the final CNN model training with VWCE loss function on fusion masks. Our evaluations are all conducted on $\mathrm{CNN}$ trained in this stage.

\section{Training}

\section{Data augmentation}

The ROIs of the pathological kidneys were cropped from the original images. The size of ROI is fixed at $150 * 150 * N$. Due to limited memory of GPU, the original ROIs were resampled to $128^{*} 128^{*} 64$ before fed into the network. For each data, random crops and flipping were used for data augmentation. After data augmentation, the original 120 CT images were augmented into 14,400 images for the $\mathrm{CNN}$ training.

\section{Parameter settings}

The input are ROIs of kidneys and bounding boxes without any other annotations. Considering that UNet [32] has been widely used for medical image segmentation, we adopted UNet to be the CNN models in stage2 and stage 3 in our experiments. The network parameters are updated by means of the back-propagation algorithm using the Adam optimizer. The initial learning rate was set to be 0.001 and decreased by decayed_learning_rate

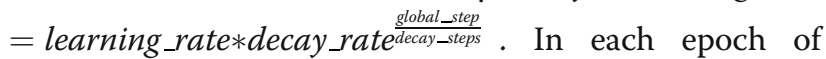

training, it takes 3600 iterations to traverse all the training images with the batch size of 4 . The class weights of cross-entropy $w_{\mathrm{c}}$ in Eqs. (8) and (9) were set to 1.0 and 0.2 for renal tumor and background respectively.

In stage2, we set the number of subset $K$ to 3 for the training dataset of $120 \mathrm{CT}$ images. Each subset contains $40 \mathrm{CT}$ images. Three $\mathrm{CNN}$ models were trained to generate corresponding predictions of each training image. And fusion masks were generated by these predictions. The loss used in this stage is WCE loss defined in Eq. (8).

In stage3, the final CNN is trained by fusion masks as weak annotation labels. We evaluated the performance of the final $\mathrm{CNN}$ model with 80 patient images. In order to remove some misclassified outlier voxels, a connected component analysis with an 18connectivity in 3D was carried out finally. The largest connected component in the output of the final CNN model was extracted as the segmentation results of renal tumors.

\section{Existing methods}

We mainly compared with two weakly-supervised methods, i.e., SDI [5] and constrained-CNN [21]. The SDI method used 2D UNet to generate weak labels from bounding box by recursive training and carry out final segmentation. The weakly-supervised information used in the constrained-CNN method includes scribbles and the volume of target tissue. In this paper, the scribbles annotations used in constrained$\mathrm{CNN}$ were generated by employing binary erosion on ground truth for every slice. Furthermore, the volumetric threshold of renal tumor was used in the loss function of Constrained-CNN. It was set to $[0.9 \mathrm{~V}$, $1.1 \mathrm{~V}$, where $V$ represents the volume of renal tumor in ground truth. As the architecture of UNet was used in $[5,21]$, as well as our proposed method, the UNet was trained by all the training dataset with the pixel-wise labels to generate a fully-supervised UNet model for extensive comparison.

\section{Results}

Our method has been implemented using PyTorch framework in version 1.1.0. The network training and testing experiments were performed on a workstation with: CPU of i7-5930K, 128GB RAM and a GPU card of NVIDIA TITAN Xp of 12GB memory.

\section{The comparison of different weak labels and training losses}

As shown in Table 1, DSCs between the different masks and the ground truth of the training dataset are displayed. The DSCs of bounding boxes are 0.666, 0.466 and 0.341 respectively when the margins of 
Table 1 DSCs between different weak labels and ground truths of the training dataset

\begin{tabular}{llll}
\hline & Bounding boxes & Pseudo masks & Fusion masks \\
\hline$d=0$ & 0.666 & 0.862 & $\mathbf{0 . 8 7 4}$ \\
$d=5$ & 0.466 & 0.801 & $\mathbf{0 . 8 1 0}$ \\
$d=10$ & 0.341 & 0.679 & $\mathbf{0 . 6 9 1}$
\end{tabular}

bounding box were set to 0,5 and 10 pixels. The DSCs of pseudo masks generated by ConvCRFs can reach 0.862, 0.801 and 0.679. However, the DSCs of fusion masks generated after group training has even higher DSC than pseudo masks. Obviously, the rectangular bounding boxes were improved significantly by the Stage 1 and Stage 2 .

Furthermore, the improvements of the weak labels contribute to the training of the final $\mathrm{CNN}$ model. Figure 6 shows the training loss of the final $\mathrm{CNN}$ model with different parameters. Without group training, the training loss shows the slowest rate and the highest loss value during training. Contrarily, the usage of group training and VWCE loss makes the model converges faster and better.

\section{Evaluation of segmentation results of renal tumors in the testing dataset with different parameters}

The DSC, Hausdorff distance (HD) [33] and average surface distance (ASD) were adopted to evaluate the segmentation results of our proposed method. The segmentation results of renal tumors in the testing dataset were obtained with different settings of parameters, i.e. number of groups, loss function and margin of bounding box. The comparison of DSCs in the testing dataset is displayed in Table 2. $k=0$ means that the procedure of stage 2 not used. In this situation, the pseudo masks generated by ConvCRFs were used as weak labels directly for the final $\mathrm{CNN}$ model training in the stage3. The loss functions used during the final model training is marked in the parentheses. MC represents the connected component analysis in the postprocessing step.

\section{The impact of group training}

According to the values in Table 2, group training can effectively improve the DSC. The DSCs increased by 3.4 , 5.1 and $2.5 \%$ when the margin of bounding box was set to 0,5 and 10 pixels respectively.

\section{The impact of VWCE loss}

The usage of VWCE loss made further improvement of the DSC. The DSCs increased by $1.2,3.6$, and $2.1 \%$ respectively when the margin of bounding box was set to 0,5 and 10 pixels. In addition, the application of VWCE loss and MC can alleviate the outliers in the segmentation result. The values of HD and ASD decreased significantly. Finally, the highest DSCs of $0.834,0.826$ and 0.742 can be achieved respectively when different margins of bounding box were set.

Figure 7 Shows the 2D visualization of segmentation results with different parameters. Obviously, renal tumors cannot be segmented precisely without group training as shown in Fig. 7(a). With the application of group training, the over- or under-segmentation of tumors is significantly improved (Fig. 7b). However, the segmentations of the boundary are still imprecise. With the application of group training and VWCE loss function, the best segmentation results have been obtained as shown in Fig. 7(c)

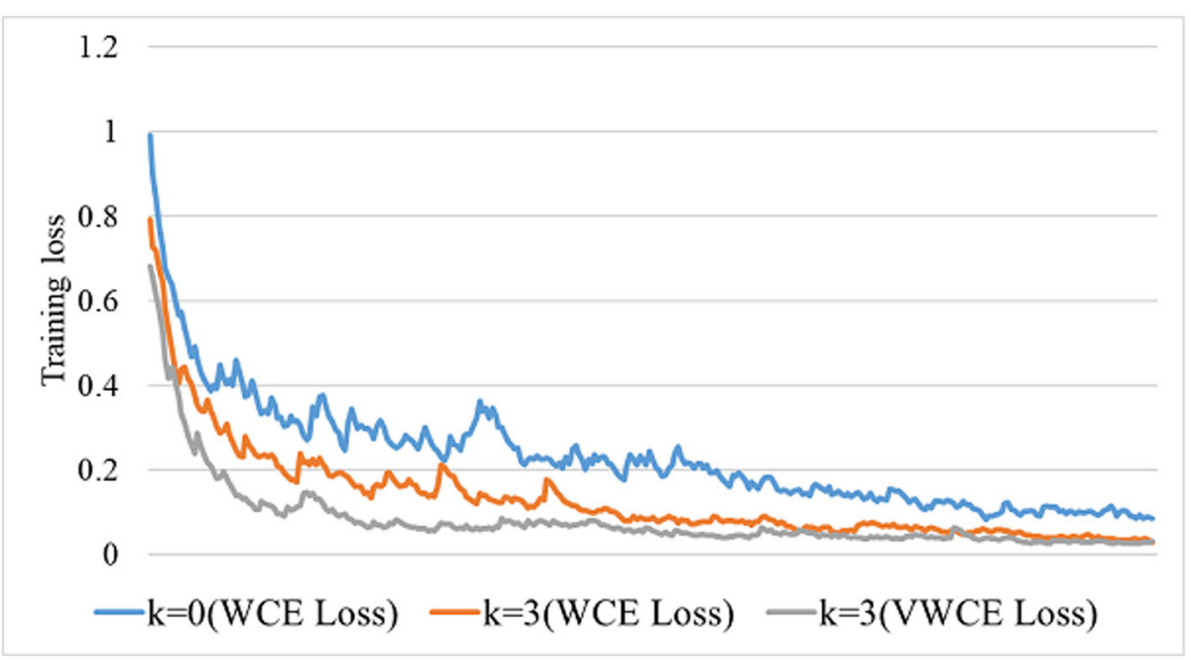

Fig. 6 Training losses of the final CNN model in stage 3 with different parameters 
Table 2 Comparison of segmentation results of testing dataset with different margins

\begin{tabular}{|c|c|c|c|c|}
\hline & & DSC & $\mathrm{HD}$ & ASD \\
\hline \multirow[t]{4}{*}{$d=0$} & $k=0$ (WCE Loss) & 0.788 & 65.806 & 6.265 \\
\hline & $k=3$ (WCE LOSS) & 0.822 & 34.187 & 3.889 \\
\hline & $k=3$ (VWCE Loss) & 0.834 & 40.617 & 3.361 \\
\hline & $k=3$ (VWCE Loss) + 3D MC & 0.834 & 14.346 & 2.664 \\
\hline \multirow[t]{4}{*}{$d=5$} & $k=0$ (WCE LosS) & 0.733 & 32.459 & 5.332 \\
\hline & $k=3$ (WCE LOSS) & 0.784 & 70.948 & 7.988 \\
\hline & $k=3$ (VWCE Loss) & 0.820 & 37.633 & 3.879 \\
\hline & $k=3$ (VWCE LosS) + 3D MC & 0.826 & 15.811 & 2.838 \\
\hline \multirow[t]{4}{*}{$d=10$} & $k=0$ (WCE LOSS) & 0.695 & 58.286 & 7.499 \\
\hline & $k=3$ (WCE Loss) & 0.720 & 81.611 & 7.804 \\
\hline & $k=3$ (VWCE Loss) & 0.741 & 36.127 & 4.672 \\
\hline & $k=3$ (WWCE Loss) + 3D MC & 0.742 & 21.233 & 4.350 \\
\hline
\end{tabular}

The DSC of each case in the testing dataset with different parameters is shown in Fig. 8. For testing dataset, it can be seen that our three-stage training strategy with VWCE loss has significantly improved the segmentation results in most images and achieves the best improvement of DSC.

\section{Comparison with other methods}

Three methods including two weakly-supervised methods (SDI and constrained-CNN) and one fullysupervised method (UNet) were used to compare with our proposed method. These methods are briefly summarized in Existing methods section. For model training, the computation time of our proposed method is about $48 \mathrm{~h}$, the SDI method is about $80 \mathrm{~h}$, and the constrained$\mathrm{CNN}$ and fully-supervised UNet are about $24 \mathrm{~h}$. for model testing, the computation time of our proposed method is similar to the fully-supervised method. Our network can generate the segmentation result of a single image in a few seconds

Table 3 is the comparison of segmentation results among our method, the other two existing weaklysupervised methods and fully-supervised method. We only compared the bounding box with $d=5$ for simplicity. Experiments show that our method achieves the best results of DSC, HD and ASD, which are 0.826, 15.811 and 2.838 respectively. In terms of DSC, neither SDI nor Constrained-CNN reaches the values higher than 0.8. One thing worth to be mentioned is that the evaluation metrics are not improved effectively in SDI after MC since we deal with it in 2D situation. When the margin is lower than 5 , the performance of our
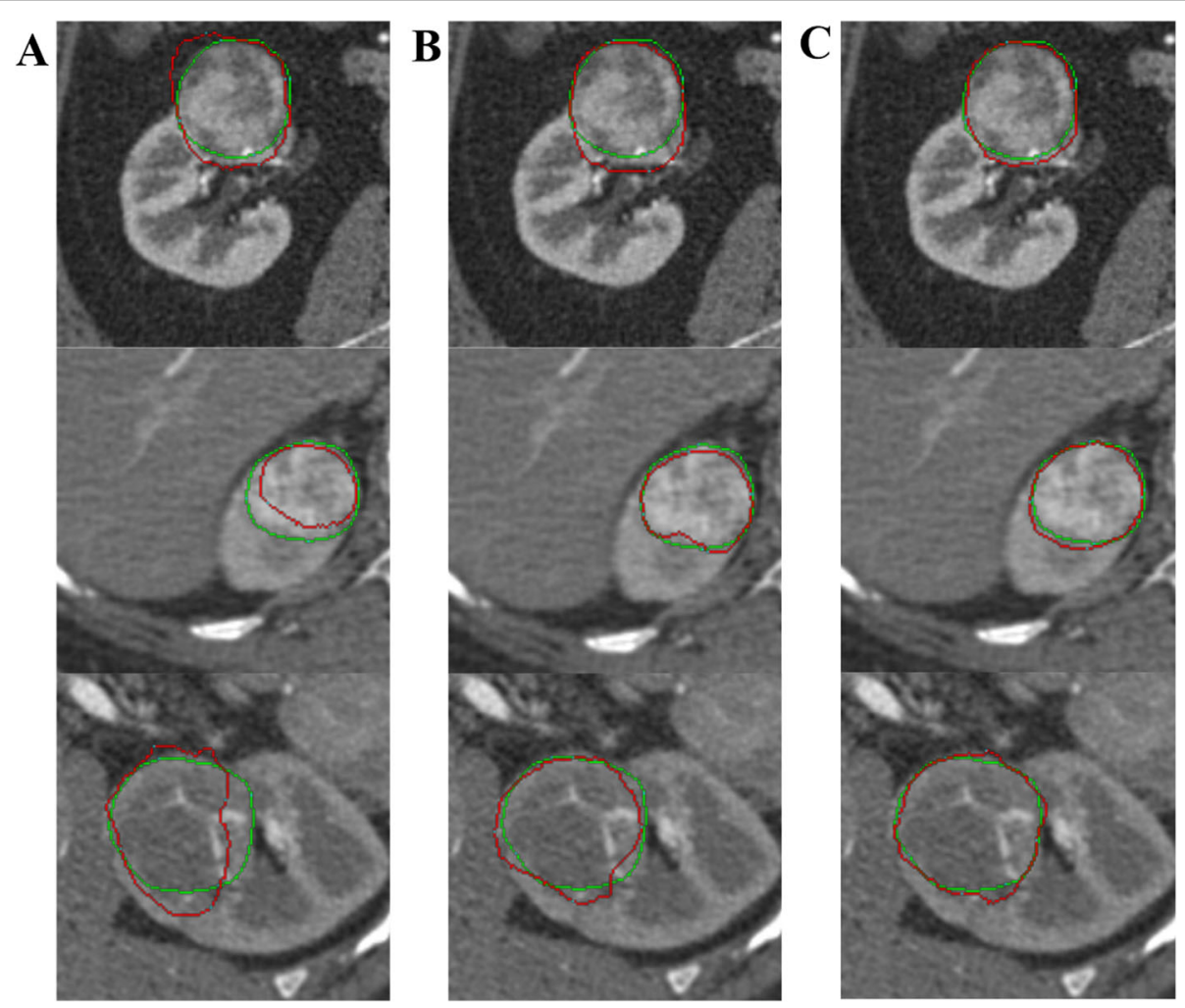

Fig. 7 The comparison of 2D segmentation results with different parameters: $k=0$ with WCE loss (a), $k=3$ with WCE loss (b), $k=3$ with WWCE loss (c). Contours in green and red correspond to ground truths and segmentation results respectively 


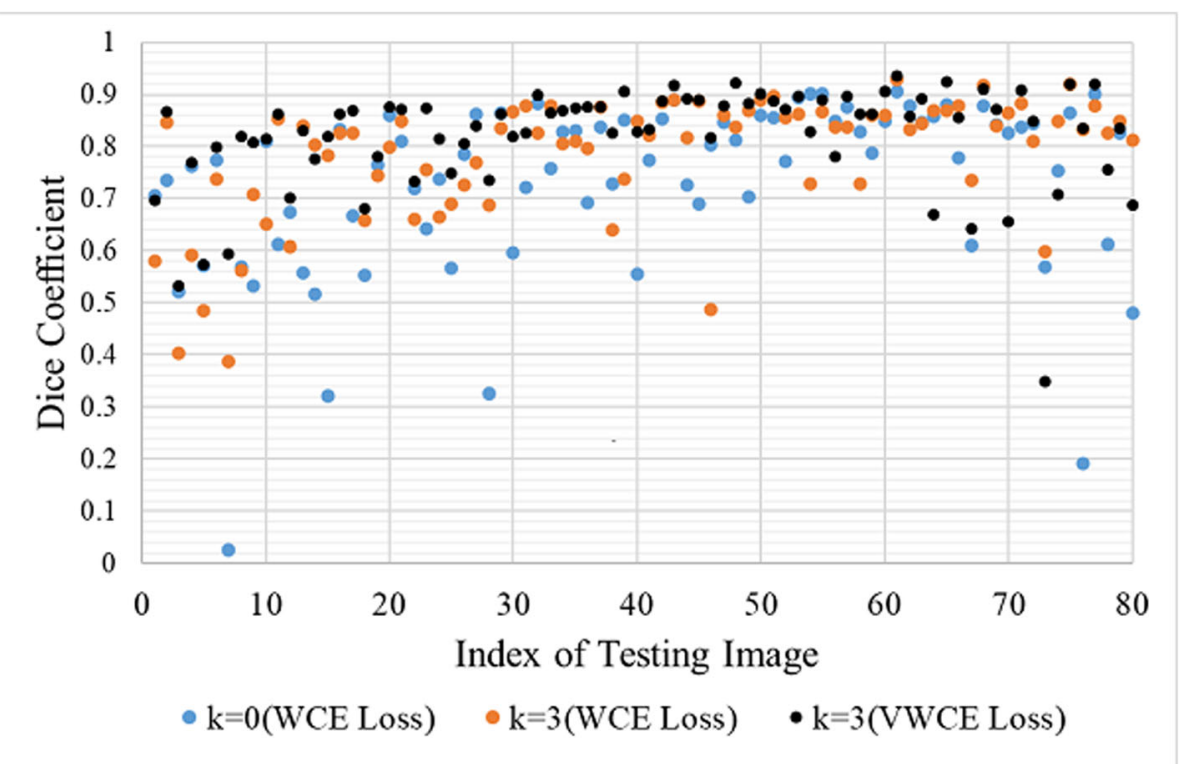

Fig. 8 DSC of each case in the testing dataset with different parameters. The index of images is ranked according to the volume of renal tumors

method is close to the results obtained by the fullysupervised UNet.

Figure 9 shows the comparison of segmentation results obtained by different methods. For SDI method, the shape of the segmented renal tumor in $3 \mathrm{D}$ is not continuous as shown in Fig. 9(b). Furthermore, SDI and Constrained-CNN still suffer from the undersegmentation problem. While, our proposed method (d) presents better segmentation results which are similar to the fully-supervised method (e) in visual.

\section{Discussion}

According to our experimental results, our proposed weakly-supervised method can provide accurate renal tumor segmentation. The major difficulty for weaklysupervised methods is that feature maps learned by CNN models can be misled by under- or oversegmentation in the weak masks. Therefore, the key

Table 3 Comparison of testing results with different methods

\begin{tabular}{llll}
\hline & DSC & HD & ASD \\
\hline Constrained-CNN [21] & 0.705 & 102.178 & 8.271 \\
Constrained-CNN [21] + 3D MC & 0.712 & 20.939 & 5.493 \\
SDI [5] & 0.766 & 73.514 & 4.639 \\
SDI [5] + 2D MC & 0.766 & 72.368 & 4.524 \\
Ours ( $d=5)$ & 0.820 & 37.633 & 3.879 \\
Ours (d=5) + 3D MC & $\mathbf{0 . 8 2 6}$ & $\mathbf{1 5 . 8 1 1}$ & $\mathbf{2 . 8 3 8}$ \\
UNet [32] (Fully-supervised) & 0.849 & 84.69 & 4.886 \\
UNet [32] (Fully-supervised) + 3D MC & 0.859 & 14.252 & 2.048 \\
\hline
\end{tabular}

factor in weakly-supervised segmentation is to generate reliable masks from the input weak labels. In this paper, the application of pseudo masks generation and group training improve the quality of the weak masks used for the final $\mathrm{CNN}$ model training as shown in Tables 1 and 2.

Furthermore, as shown in Fig. 8, the DSCs of large and small tumors are relatively low. It is easy to understand that the DSCs of the small renal tumors are sensitive to the over- or under-segmentation in the predictions. While in large tumor, the shape and texture of the tumor are complicated, which leads to the difficulties of the segmentation. Although this problem exists in all three methods, our proposed method shows the most significant improvement compared with the other two methods.

Finally, one limitation of this study is the lack of validation of the final $\mathrm{CNN}$ model with external datasets. The training and testing datasets in this paper are from the same hospital. Additional validation of the final CNN model with multi-center or multivendor images will be performed in the future. Due to the differences in image acquisition protocols or the other factors, the CNN model trained in this paper may not be able to achieve a similar performance on the other datasets. However, the parameters in our model can be optimized by fine-tuning with the external datasets to improve the accuracy. In particular, the main advantage of our method is the use of weak labels for network training, which does not take much time for radiologists to generate boundingbox labels. 

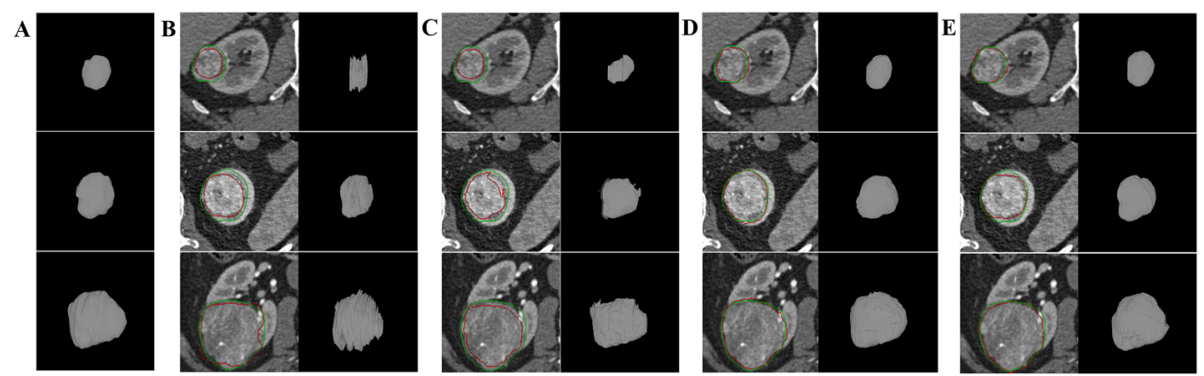

Fig. 9 The comparison of the results from three testing images obtained by different methods: 3D ground truth (a), SDI (b), Constrained-CNN(c), the proposed method (d) and fully-supervised method (e). Contours in green and red correspond to ground truth and segmentation results respectively

\section{Conclusion}

In this paper we have presented a novel three-stage training method for weakly supervised CNN to obtain precise renal tumor segmentation. The proposed method mainly relies on the group training and weighted training phases to improve not only the efficiency of training but also the accuracy of segmentation. Experimental results with 200 patient images show that the DSCs between ground truth and segmentation results can reach $0.834,0.826$ when the margin of bounding box was set to 0 and 5 , which are close to the fully-supervised model which is 0.859 . The comparison between our proposed method and the other two existing methods also demonstrate that our method can generate a more accurate segmentation of renal tumors than the other two methods.

\section{Abbreviations}

ASD: Average surface distance; CE: Cross-entropy; CNN: Convolutional neural network; ConvCRFs: Convolutional conditional random fields;

CRF: Conditional random field; CT: Computed tomography; CTA: Computed tomographic angiography; DSC: Dice coefficient; FCN: Fully convolutional network; HD: Hausdorff distance; LPN: Laparoscopic partial nephrectomy; MAP: Maximum a posteriori; MC: Maximum connected component; MR: Magnetic resonance; RCC: Renal cell carcinoma; ROI: Region of interest; SVM: Support vector machine; WWCE: Voxel-wise weighted cross-entropy; WCE: Weighted cross-entropy

\section{Acknowledgements}

We acknowledge Key Laboratory of Computer Network and Information Integration, Southeast University, Ministry of Education, Nanjing, People's Republic of China for providing us the computing platform.

\section{Authors' contributions}

GYY and CXW designed the proposed method and implemented this method. LT and PFS outlined the data label. JY, YC, JLD, HZS and LML performed the experiments and the analysis of the results. All authors have been involved in drafting and revising the manuscript and approved the final version to be published. All authors read and approved the final manuscript.

\section{Funding}

This study was funded by a grant from the National Key Research and Development Program of China (2017YFC0107900), National Natural Science Foundation $(31571001,61828101)$, Key Research and Development Project of Jiangsu Province (BE2018749) and the Southeast University-Nanjing Medical University Cooperative Research Project (2242019K3DN08). These funds provided financial support for the research work of our article but had no role in the study.

\section{Availability of data and materials}

The clinical data and materials used in this paper are not open to public, but are available from the corresponding author on reasonable request.

\section{Ethics approval and consent to participate}

This study was carried out in accordance with the recommendations of name of the Nanjing Medical University's Committee with written informed consent from all subjects. All subjects gave written informed consent in accordance with the Declaration of Helsinki. The protocol was approved by the name of the Nanjing Medical University's Committee.

\section{Consent for publication}

Not applicable.

\section{Competing interests}

Yang Chen, one of the co-authors, is a member of the editorial board (Associate Editor) of this journal. The other authors have no conflicts of interest to disclose.

\section{Author details}

'LIST, Key Laboratory of Computer Network and Information Integration, Southeast University, Ministry of Education, Nanjing, China. ${ }^{2}$ Centre de Recherche en Information Biomédicale Sino-Français (CRIBs), Rennes, France. ${ }^{3}$ Beijing Engineering Research Center of Mixed Reality and Advanced Display, School of Optics and Electronics, Beijing Institute of Technology, Beijing 100081, China. ${ }^{4}$ Department of Radiology, The First Affiliated Hospital of Nanjing Medical University, Nanjing, China. ${ }^{5}$ Department of Urology, The First Affiliated Hospital of Nanjing Medical University, Nanjing, China. ${ }^{6}$ University Rennes, Inserm, LTSI - UMR1099, F-35000 Rennes, France.

Received: 10 December 2019 Accepted: 20 March 2020

Published online: 15 April 2020

\section{References}

1. Ljungberg B, Bensalah K, Canfield S, Dabestani S, Hofmann F, Hora M, et al. EAU guidelines on renal cell 569 carcinoma 2014 update. Eur Urol. 2015; 67(5):913-24.

2. Litjens GJ, Kooi T, Bejnordi BE, Setio AA, Ciompi F, Ghafoorian M, et al. A survey on deep learning in medical image analysis. Med Image Anal. 2017; 42:60-88.

3. Dai J, He K, Sun J. BoxSup: exploiting bounding boxes to supervise convolutional networks for semantic segmentation. In: The IEEE International Conference on computer vision; 2015. p. 1635-43.

4. Papandreou G, Chen L, Murphy K, Yuille AL. Weakly-and semi-supervised learning of a deep convolutional network for semantic image segmentation. In: The IEEE International conference on computer vision; 2015. p. 1742-50

5. Khoreva A, Benenson R, Hosang J, Hein M, Schiele B. Simple does it: weakly supervised instance and semantic segmentation. In: The IEEE conference on computer vision and pattern recognition; 2017. p. 876-85. 
6. Hu R, Dollar P, He K, Darrell T, Girshick R. Learning to segment everything. In: The IEEE Conference on computer vision and pattern recognition; 2018 p. 4233-41.

7. Tang M, Djelouah A, Perazzi F, Boykov Y, Schroers C. Normalized cut loss for weakly-supervised CNN segmentation. In: The IEEE Conference on computer vision and pattern recognition; 2018. p. 1818-27.

8. Lin D, Dai J, Jia J, He K, Sun J. ScribbleSup: scribble-supervised convolutional networks for semantic segmentation. In: The IEEE Conference on computer vision and pattern recognition; 2016. p. 3159-67.

9. Maninis K, Caelles S, Ponttuset J, Gool L. Deep extreme cut: from extreme points to object segmentation. In: The IEEE Conference on computer vision and pattern recognition; 2018. p. 616-25.

10. Bearman A, Russakovsky O, Ferrari V, Fei-Fei L. What's the point: semantic segmentation with point supervision. In: European Conference on computer vision; 2016. p. 549-65.

11. Pathak D, Shelhamer E, Long J, Darrell T. Fully convolutional multi-class multiple instance learning. 2014; arXiv: 1412.7144.

12. Pinheiro PO, Collobert R. From image-level to pixellevel labeling with convolutional networks. In: The IEEE Conference on computer vision and pattern recognition; 2015. p. 1713-21.

13. Saleh FS, Aliakbarian MS, Salzmann M, Petersson L, Gould S, Alvarez JM. Built-in foreground/background prior for weakly-supervised semantic segmentation. In: European Conference on Computer Vision; 2016. p. 413-32.

14. Wei $Y$, Liang $X$, Chen $Y$, Shen X, Cheng M, Feng J, et al. STC: a simple to complex framework for weakly-supervised semantic segmentation. IEEE Trans Pattern Anal Mach Intell. 2017;39(11):2314-20.

15. Kolesnikov A, Lampert CH. Seed, expand and constrain: three principles for weakly-supervised image segmentation. In: European conference on computer vision; 2016. p. 695-711.

16. Qi X, Liu Z, Shi J, Zhao H, Jia J. Augmented feedback in semantic segmentation under image level supervision. In: European conference on computer vision; 2016. p. 90-105.

17. Wei $Y$, Feng J, Liang $X$, Cheng M, Zhao $Y$, Yan S. Object region mining with adversarial erasing: a simple classification to semantic segmentation approach. In: The IEEE Conference on computer vision and pattern recognition; 2017. p. 1568-76.

18. Rajchl M, Lee MC, Oktay O, Kamnitsas K, Passerat-Palmbach J, Bai W, et al. DeepCut: object segmentation from bounding box annotations using convolutional neural networks. IEEE Trans Med Imaging. 2017;36(2):674-83.

19. Rajchl M, Lee MC, Schrans F, Davidson A, Passerat-Palmbach J, Tarroni G, et al. Learning under distributed weak supervision. 2016; arXiv: 1606.01100

20. Achanta R, Shaji A, Smith K, Lucchi A, Fua P, Süsstrunk S. Slic superpixels compared to state-of-the-art superpixel methods. IEEE Trans Pattern Anal Mach Intell. 2012;34(11):2274-82.

21. Kervadec H, Dolz J, Tang M, Granger E, Boykov Y, Ayed IB. Constrained-CNN losses for weakly supervised segmentation. Med Image Anal. 2019;54:88-99.

22. Linguraru MG, Yao J, Gautam R, Peterson J, Li Z, Linehan WM, et al. Renal tumor quantification and classification in contrast-enhanced abdominal CT. Pattern Recogn. 2009;42(6):1149-61.

23. Linguraru MG, Wang S, Shah F, Gautam R, Peterson J, Linehan WM, et al. Automated noninvasive classification of renal cancer on multiphase CT. Med Phys. 2011;38(10):5738-46

24. Yang G, Li G, Pan T, Kong Y, Wu J, Shu H, et al. Automatic segmentation of kidney and renal tumor in CT images based on 3D fully convolutional neural network with pyramid pooling module. In: International Conference on pattern recognition; 2018. p. 3790-5.

25. Yu Q, Shi Y, Sun J, Gao Y, Zhu J, Dai Y. Crossbar-net: a novel convolutional neural network for kidney tumor segmentation in CT images. IEEE Trans Image Process. 2019;28(8):4060-74.

26. Zhang J, Lefkowitz RA, Ishill NM, Wang L, Moskowitz CS, Russo P, et al. Solid renal cortical tumors: differentiation with CT. Radiology. 2007;244(2):494504.

27. Lin TY, Maire M, Belongie S, Hays J, Perona P, Ramanan D, et al. Microsoft coco: common objects in context. In: European Conference on computer vision; 2014. p. 740-55

28. Wang X, You S, Li X, Ma H. Weakly-supervised semantic segmentation by iteratively mining common object features. In: The IEEE Conference on computer vision and pattern recognition; 2018. p. 1354-62.

29. Yang G, Gu J, Chen Y, Liu W, Tang L, Shu H, et al. Automatic kidney segmentation in $C T$ images based on multi-atlas image registration. In:
Annual International Conference of the IEEE engineering in medicine and biology society; 2014. p. 5538-41.

30. Teichmann M, Cipolla R. Convolutional CRFs for semantic segmentation. 2018; arXiv: 1805.04777.

31. Krahenbuhl P, Koltun V. Efficient inference in fully connected CRFs with Gaussian edge potentials. In: Advances in neural information processing systems; 2011. p. 109-17.

32. Ronneberger O, Fischer P, Brox T. U-Net: convolutional networks for biomedical image segmentation. In: International conference on medical image computing and computer assisted intervention; 2015. p. 234-41.

33. Huttenlocher DP, Klanderman GA, Rucklidge WJ. Comparing images using the Hausdorff distance. IEEE Trans Pattern Anal Mach Intell. 1993;15(9):85063.

\section{Publisher's Note}

Springer Nature remains neutral with regard to jurisdictional claims in published maps and institutional affiliations.
Ready to submit your research? Choose BMC and benefit from:

- fast, convenient online submission

- thorough peer review by experienced researchers in your field

- rapid publication on acceptance

- support for research data, including large and complex data types

- gold Open Access which fosters wider collaboration and increased citations

- maximum visibility for your research: over $100 \mathrm{M}$ website views per year

At BMC, research is always in progress.

Learn more biomedcentral.com/submissions 